\title{
Effects of Transcranial Magneto-acoustic Electrical Stimulation on Discharge propagation in feed- forward neural network
}

Jiankang Wu (D 13116127056@163.com )

hebei university of technology https://orcid.org/0000-0002-3997-211X

Shuai Zhang

Hebei University of Technology

Jiayue Xu

Hebei University of Technology

Junwu Dang

Hebei University of Technology

Qingyang Zhao

Hebei University of Technology

Mingkang Zhao

Hebei University of Technology

Guizhi Xu

Hebei University of Technology

Xueying Zhang

Hebei University of Technology

Hongbin Wang

Hebei University of Technology

Wenjing Du

Hebei University of Technology

\section{Research Article}

Keywords: Transcranial magneto-acoustic electrical stimulation, feed-back, Izhikevich, electrical coupling

Posted Date: January 12th, 2022

DOI: https://doi.org/10.21203/rs.3.rs-1234073/v1

License: (1) (1) This work is licensed under a Creative Commons Attribution 4.0 International License.

Read Full License 


\title{
Effects of Transcranial Magneto-acoustic Electrical Stimulation on
}

\section{Discharge propagation in feed-forward neural network}

\author{
Jiankang $\mathrm{Wu}^{1.2}$ Shuai Zhang ${ }^{1.2^{*}}$ Jiayue $\mathrm{Xu}^{1.2}$ Xueying Zhang ${ }^{1.2}$ Junwu Dang ${ }^{1.2}$ Wenjing Du ${ }^{1.2}$ Qingyang Zhao ${ }^{1.2}$ \\ Mingkang Zhao ${ }^{1,2}$ Hongbin Wang ${ }^{1.2}$ Guizhi $\mathrm{Xu}^{1.2}$
}

(1 State Key Laboratory of Reliability and Intelligence of Electrical Equipment, Hebei University of Technology, Tianjin 300130, China (2 Tianjin Key Laboratory of Bioelectricity and Intelligent Health, Hebei University of Technology, Tianjin 300130, China

\begin{abstract}
The mammalian brain has an extremely complex, diversified and highly modular structure, and information dissemination in the modular brain network affects various brain diseases. Although a variety of neuromodulation techniques have been used to study the discharge characteristics of neural networks, the effects of transcranial magneto-acoustic electrical stimulation(TMAES) have rarely been mentioned. Based on the excitatory and inhibitory Izhikevich neuron model, we constructs a feed-forward neural network connected by electrical synapses and chemical synapses, and analyzes the firing frequency of the neural network under TMAES and magnetic stimulation and the differences in each layer types of firing patterns of neurons. The results showed that the discharge patterns of neurons in each layer were different, the discharge frequency of inhibitory neurons was higher than that of excited neurons, and the stimulation signal could be transmitted to the entire network layer.The maximum discharge frequency of neural network connected by electrical coupling can reach $0.94 \mathrm{kHz}$, and the discharge frequency of neural network connected by chemical coupling is less than $0.5 \mathrm{kHz}$. With the increase of the coupling degree, the discharge frequency of neurons in each network layer under TMAES is much greater than that under magnetic stimulation. When the induced current is lower than $26.5 \mu \mathrm{A} / \mathrm{cm}^{2}$, magnetic stimulation can promote the inhibitory neurons, and TMAES has a variety of regulatory effects on the inhibitory neurons in the neural network. The results show that TMAES has better regulation effect than magnetic stimulation, and the regulation effect is affected by a neural network structure and stimulation parameters.
\end{abstract}

Keywords: Transcranial magneto-acoustic electrical stimulation, feed-back, Izhikevich, electrical coupling 


\section{Introduction}

The nervous system is a highly modular structure with strong information processing ability. The information processing in the nervous system is related to different functional groups of neurons. Through these functional groups, information is transferred from one group to its downstream connection group ${ }^{[1]}$. Pulse activity affects neural information coding ${ }^{[2]}$.Abnormal information coding will lead to brain dysfunction and lead to brain functional diseases such as Parkinson's disease, epilepsy and depression ${ }^{[3]}$.Different stimuli will produce different responses to information encoding, which plays an important role in the realization of advanced functions of the $\operatorname{brain}^{[4]}$.Transcranial magneto-acoustic electrical stimulation (TMAES) is a new non-invasive technique for brain stimulation and regulation. It is different from transcranial direct current stimulation, magnetic stimulation and transcranial ultrasound stimulation ${ }^{[5-9]}$. By using ultrasound and static magnetic field to act on nerve tissue, it can produce induced current in nerve tissue and regulate nerve activity in the corresponding brain regions. However, few scholars have studied the influence of TMAES on neural network information coding.

Existing scholars have analyzed the time coding characteristics of SFNN under different stimulation intensities such as Gaussian stimulation, pulse stimulation, and $\mathrm{AC}$ electric field stimulation, and found that the stronger the stimulation intensity, the smaller the ISI; the time coding is specific; the dynamic adjustment of synaptic plasticity is time Intrinsic factors of the coding mechanism ${ }^{[10]}$.In addition, the Izhikevich network with excitatory and inhibitory synapses was constructed, and it was found that inhibitory synapses could not only prevent synchronization by delaying the discharge of neurons, but also promote synchronization by promoting the transformation of oscillation modes ${ }^{[11]}$.Eteme et al. found that magnetic stimulation not only induces spikes and bursts in regular neuronal activity, but also induces synchronous firing of neurons ${ }^{[12]}$.Tan et al. considered the complex interaction between the intrinsic properties of neurons and the network structure in the propagation of impulses. Different neuronal ratios will affect the propagation of impulses ${ }^{[13]}$.However, in the study of FFN, the influence of TMAES parameters on the discharge characteristics of FFN is rarely considered, and whether the internal electrical characteristics of different coupling types of neurons are considered in the discharge behavior propagating in FFN.

Currently commonly used neuron models include $\mathrm{H}-\mathrm{H}$ neuron model, chay neuron model, ML neuron model and Izhikevich neuron model ${ }^{[14-17]}$. The Izhikevich neuron model has the electrophysiological characteristics of the $\mathrm{HH}$ neuron model and the computational efficiency of the ML neuron model. Suitable for building neural networks on a large scale $^{[18]}$. Neurosynapses are divided into electrical synapses and chemical synapses ${ }^{[19]}$. Electrical synapses connect with each other through low-voltage ion channels formed by the combination of proteins. The signal transmission is bidirectional ${ }^{[20]}$. Chemical synapses need to release neurotransmitters through presynaptic neuron units. Sensors in the post synaptic membrane line accept neurotransmitters and do not have bidirectionality ${ }^{[21]}$.

The main contributions of this paper are as following. Based on the Izhikevich neuron model, the specificity of information encoding of neural networks with different structures under transcranial magneto-acoustic stimulation, magnetic field stimulation and direct current stimulation is studied, and the information transfer efficiency of neural networks is considered under different coupling forms.

The rest of this study is arranged as follows. In the second part, a feed-forward neural network is constructed. The third part focuses on the coding information of feed-forward neural network under different stimuli. The fourth part focuses on the conclusion.

\section{Model and Methods \\ 2.1 Principle of TMAES}


Transcranial magneto-acoustic electrical stimulation is a neural regulation method that combines static magnetic field and ultrasound to stimulate organisms. There are charged ions in biological tissues, and the ions in biological tissues will shift due to ultrasonic vibration after applying ultrasound ${ }^{[22]}$. Under the action of a static magnetic field, these charged ions will deflect under the action of the Lorentz force, resulting in the induced current. Through the combined effect of ultrasound and induced current, the activity of neurons and their discharge rhythm can be affected to achieve effective regulation of the nervous system.

The induced current generated by magneto-acoustic stimulation on nerve tissue is :

$$
I_{e x t}=\sigma B \sqrt{\frac{2 \Gamma}{\rho c_{0}}}
$$

Where $\Gamma$ denotes the ultrasonic intensity, $\rho$ denotes the tissue density, and $c_{0}$ denotes the propagation velocity of ultrasound in biological tissues. $f$ means ultrasonic frequency, B means magnetic induction intensity of static magnetic field in magneto-acoustic stimulation, $\sigma$ means electrical conductivity of nervous tissue.

\subsection{Neuron model}

Eugene M.Izhikevich established a simple pulsed neuron model by combining the biodynamics of the Hodgkin-Huxley neuron model and the computational efficiency of IF neuron model, which can reproduce the electrical activity of a large number of cortical neurons. Its dynamic equation is as following:

$$
\begin{gathered}
\frac{d v}{d t}=0.04 v^{2}+5 v+140-u+I_{e x t} \\
\frac{d u}{d t}=a(b v-u)
\end{gathered}
$$

With the auxiliary after spike resetting:

If $v \geq 30 \mathrm{mV}$

$$
\left\{\begin{array}{l}
v \leftarrow c \\
u \leftarrow u+d
\end{array}\right.
$$

Here, $v$ represents the neuronal membrane potential, $u$ represents the membrane recovery variable, and provides negative feedback to the membrane potential. The neuron model parameter $a$ represents the recovery constant, $b$ describes the susceptibility of the recovery variable to the subthreshold fluctuation of membrane potential, $c$ represents the reset value of membrane potential when the voltage reaches $30 \mathrm{mV}, d$ represents the reset of recovery variables.

During AC magnetic stimulation, the neuron model is shown in Equation 5.

$$
\left\{\begin{aligned}
\frac{d v}{d t}= & 0.04 v^{2}+5 v+140 \\
& -u+I-k_{1} \rho(\varphi) v \\
\frac{d u}{d t}= & a(b v-u) \\
\frac{d \varphi}{d t}= & k_{2} x-k_{3} \varphi+\varphi_{e}
\end{aligned}\right.
$$

Here, the variable $I$ represents the synaptic current, and its value is equal to $10 . \varphi_{e}=A \cos (w t)$ is the external flux applied to the neuron and $\mathrm{A}=5, \mathrm{w}=$ 0.2.The third variable $\varphi$ represents the magnetic flux. $\rho(\varphi)$ is the memory conductance, which defines the relationship between the membrane potential and magnetic flux.Membrane resistance used is flux controlled, and $\rho(\varphi)=\alpha \varphi^{2}+\beta \varphi+\gamma \quad$ represents its conductance.Here $\alpha, \beta_{\gamma}$ are constant parameters $\alpha=0.01, \beta=0.5, \gamma=0.5$. The expression of $k_{1} \rho(\varphi) v$ depends on the change of magnetic flux, which determines the current added to the right side of the membrane voltage equation.Constant parameters $\mathrm{k} 1=0.35$.

\subsection{Neural network}

There are billions of neurons in the cerebral cortex, electrical synapses and chemical synaptic interactions. The neurons in front of the synapse are called presynaptic neurons, and the neurons behind the synapse are called post synaptic neurons ${ }^{[23]}$.In chemical synapses, presynaptic neurons secrete neurotransmitters that affect post synaptic neurons. In excitatory inhibitory interaction of presynaptic neurons, the intramembrane charge is more than the extracellular charge, while the intramembrane charge of postsynaptic neurons is mostly negative ${ }^{[24]}$. That is, chemical synapses can only transmit in one direction. 
In this study, ten layers of feed-forward neural network were constructed, each layer contained five neurons, and the excitation inhibition ratio was 4:1.The neurons in the same layer are not connected, and the neurons between layers are connected by chemical coupling. In the current study, a 10-layer feed-forward network was constructed, each layer containing 200 Izhikevich neurons. There is no connection between neurons in the same layer. Each neuron from the previous layer of neurons, access to chemical synaptic input.The neural network is expressed as:

$$
\left\{\begin{array}{c}
\frac{d V_{l, i}}{d t}=0.04 V_{l, i}^{2}+5 V_{l, i}+140 \\
-u_{l, i}+I_{l, i}^{e x t}+I_{l, i}^{s y n} \\
\frac{d u_{l, i}}{d t}=a_{l, i}\left(b_{l, i} V_{l, i}-u_{l, i}\right)
\end{array}\right.
$$

Where $V_{l, i}$ represents the membrane potential of the ith neuron in layer $L$, and $u_{l}$, I represents the recovery variable of the $i$ th neuron in layer $L, I^{\mathrm{syn}}{ }_{\text {ext }}$ represents external stimulation current, $I^{s y n}{ }_{l, i}$ represents chemical synaptic current, as shown in the following formula:

$$
I_{l, i}^{s y n}=\frac{H}{1+e^{-\sigma\left(V_{l, i}-\theta\right)}} \sum_{j=1}^{N}\left(V_{s y n}-V_{l \dashv, j}\right)
$$

Where $H$ is the chemical coupling strength, $V_{s y n}$ represents synaptic reversible potential, $\sigma$ is the ratio constant, $\theta$ represents synaptic threshold. $\left(V_{s y n}=-65, \sigma\right.$ $=10, \theta=-45, \mathrm{~N}=200) . I^{s y n}$ represents electrical synaptic current, as showing in the following formula:

$$
I_{l, i}^{s y n}=g\left(V_{l, j}-V_{l-1, j}\right)
$$

Where $g$ is the electric coupling strength,$V_{l, i}$ represent the membrane potential of the ith neuron in layer $l . V_{l-1, i}$ represents the membrane potential of the $i$ th neuron in layer $l-1$.

The average discharge rate $f$ is used to represent the discharge state of neural networks with different connection structures. The definition formula is:

$$
f=\frac{1}{N} \sum_{i=1}^{N} f_{i}
$$

\section{Results}

When the feed-forward neural network is connected in the form of electrical coupling and chemical coupling, the neural signal transmission characteristics of the neural network under TMAES and TMS and the spike release rates of neurons with different properties are analyzed.

\subsection{Electrical coupling}

When the feed-forward neural network is connected in the form of electrical coupling with a coupling strength of 0.2 and a transcranial magneto-acoustic electrical stimulation current of 100 $\mu \mathrm{A} / \mathrm{cm}^{2}$, the membrane potential changes of excitatory and inhibitory neurons in each layer are shown in Figure 3.1(a) and (b). With the increase of network layer number, the spikes of excitable neurons become sparse, sub-threshold oscillation occurs, but the fifth layer network basically does not discharge. The spikes of inhibitory neurons become sparse, but they are still denser than the excitable neurons, which can transmit more coding information.As shown in Fig. 3.1 (c) and (d), Changes in membrane potential of excitatory and inhibitory neurons in feed-forward neural networks under TMS.The interpeak period of excitatory neurons in the stimulation layer was larger than that in the TMAES group under the same conditions. With the increase of the number of network layers, the number of spikes of excitatory neurons decreased.The interpeak period of inhibitory neurons in stimulation layer is less than that of excitatory neurons. With the increase of network layer, the number of discharge peaks decreases and the interpeak period increases.The spike number of neural network in TMAES group was significantly greater than that in TMS group, and the depth of neural signal transmission generated by stimulation was deeper. Compared with excited neurons, the number of spikes that inhibit neurons is more, and the depth of nerve signal transmission is deeper. 

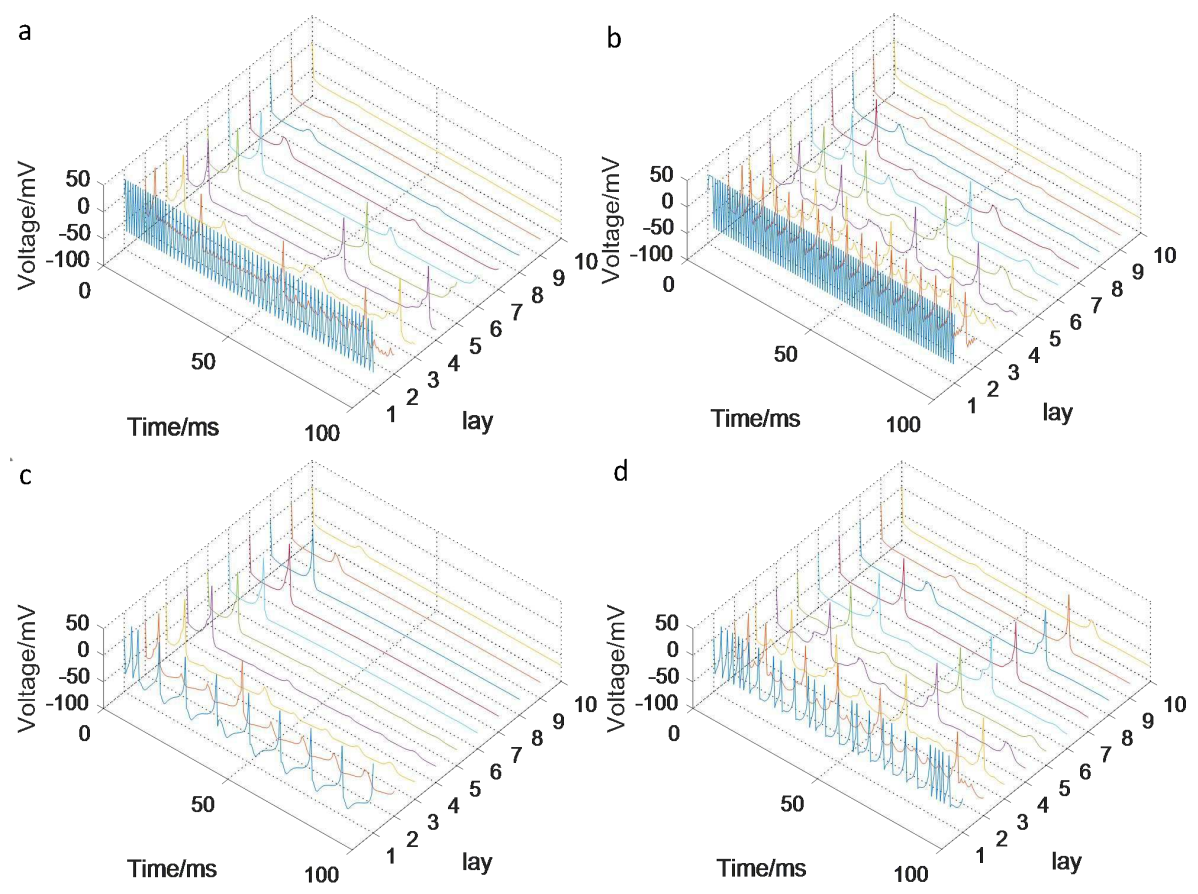

Figure 3.1 Membrane potential of neurons under different external stimuli. a)Membrane potential of excitable neurons under TMAES.b)The membrane potential of inhibitory neurons under TMAES. c)Membrane potential of excitable neurons under TMS.d)The membrane potential of inhibitory neurons under TMS.

The synapses in the feed-forward neural network are connected in the form of electrical coupling. When the induced current is $100 \mu \mathrm{A} / \mathrm{cm}^{2}$, the discharge frequency of neurons in each layer increases with the increase of electrical coupling, and decreases with the increase of layers, as shown in Fig. 3.2 (a) and Fig. 3.2 (b). When the coupling degree is $0 \sim 0.9$, the discharge frequency of excitable neurons in each layer increases with the increase of coupling degree, and the discharge frequency of the third layer and deeper network is approximate. After the electrical coupling degree is greater than 0.9 , the discharge frequency of the last three layers decreases, and the other layers maintain an upward trend. The discharge frequency of inhibitory neurons in each layer fluctuated violently with the increase of coupling degree. When the electrical coupling strength is $0.5 \sim 0.6$, the discharge frequency of the second layer neurons decreases and is approximately equal to the discharge frequency of the third layer neurons. When the electrical coupling degree is $0.6 \sim 0.9$, the discharge frequency of the second layer and the third layer is basically unchanged, and the discharge frequency of the deep network increases with the increase of the coupling degree. When the coupling degree is greater than 0.9 , the network discharge frequency of the second and third layers increases significantly, and the discharge frequency of the third layer is still smaller than that of the second layer. It shows that the discharge frequency of inhibitory neurons is easily affected by the electrical coupling strength, and it is more significant with the increase of network layers.

As shown in Fig. 3.2 (c) and Fig. 3.2 (d), Under transcranial magnetic stimulation, the firing rate curves of excitatory and inhibitory neurons in the stimulation layer of feed-forward neural network with the increase of coupling degree.The firing rate of excitatory neurons in each layer increases with the increase of coupling degree. The excitatory neurons in the fourth layer of the network start to discharge when the coupling degree is 0.3 , and no longer change when the electrical coupling degree is greater than 0.4, which is $0.16 \mathrm{kHz}$.Excited neurons in the tenth layer of feed-forward network start firing when the coupling degree reaches 0.9 , that is, when the 
coupling strength reaches a certain value, the stimulus
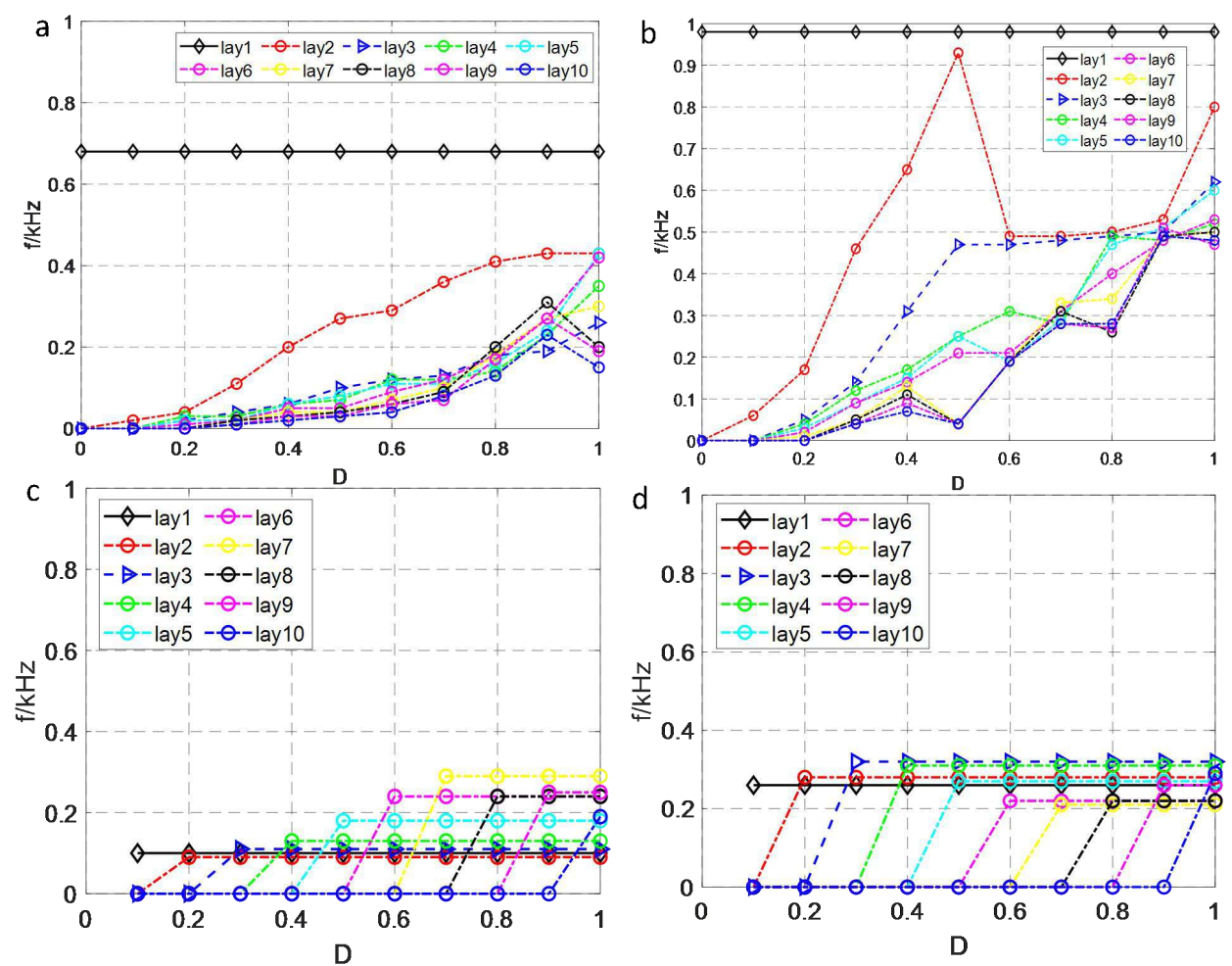

Figure 3.2 Neuron discharge frequency under different external stimuli. a)Excitable neuron discharge frequency under TMAES.b)Discharge frequency of inhibitory neurons under TMAES. c)Excitable neuron discharge frequency under TMS.d)Discharge frequency of inhibitory neurons under TMS.

can be transmitted to the tenth layer network.In addition, the seventh-layer network has the highest discharge rate, indicating that there is a coupling threshold for the stimulation depth. The firing rate of inhibited neurons in each layer increased with the increase of coupling degree, but the stable value was greater than that of excited neurons in each layer.When the degree of electrical coupling is 1 , the neurons in each layer of the feed-forward neural network are all firing, and the firing rate is about 300 Hz.This indicates that magnetic stimulation has a stronger effect on inhibitory neurons in the network

In Fig. 3.3 (a) (b) shows that the synapses in the feed-forward neural network are connected in the form of electrical coupling. When the coupling strength is 0.5 , the discharge frequency of neurons in each layer of the network increases with the induction current. The discharge frequency of excitable neurons in the stimulus layer changed linearly with the increase of the induction current. When the induction current was $100 \mu \mathrm{A} / \mathrm{cm}^{2}$, the discharge frequency reached $0.75 \mathrm{kHz}$. The discharge frequency of the second layer excitable neurons increased with the increase of the induced current; the discharge frequency of excitatory neurons in the 3 rd to 10 th layers of the network fluctuated little, basically stabilized at $0.1 \mathrm{kHz}$. With the increase of current, the discharge frequency of inhibitory neurons fluctuates greatly in each layer. When the induced current of the inhibitory neurons in the stimulus layer was less than $48 \mu \mathrm{A} / \mathrm{cm}^{2}$, the discharge frequency of the inhibitory neurons increased with the increase of the induced current. When the induced current of the inhibitory neurons in the second layer was $40 \mu \mathrm{A} / \mathrm{cm}^{2}$, the discharge frequency decreased, and showed a fluctuating upward trend in the range of $0 \mu \mathrm{A} / \mathrm{cm}^{2} \sim$ $100 \mu \mathrm{A} / \mathrm{cm}^{2}$. From the third layer to the tenth layer, the fluctuation of discharge frequency of inhibitory neurons is small, but it is greater than that of excitatory neurons, which indicates that inhibitory neurons are more susceptible to the change of induced current and more information is transmitted in the 
network.

As shown in Fig. 3.3 (c) and Fig. 3.3 (d), the variation of neuron discharge rate with induced current in each layer of feed-forward neural network under transcranial magnetic stimulation. The discharge rate of neurons in the stimulating layer increased linearly with the increase of induced current, when the induced current is greater than $30 \mu \mathrm{A} / \mathrm{cm}^{2}$, the rising trend of the discharge rate of the second layer slows down, and the discharge rate of excited neurons from the third layer to the tenth layer is basically unchanged.When the induced current was less than $22 \mu \mathrm{A} / \mathrm{cm}^{2}$, the discharge frequency increased with the increase of induced current, and reached the maximum of $0.82 \mathrm{kHz}$ when the induced current was $22 \mu \mathrm{A} / \mathrm{cm}^{2}$. When the induced current is $22 \sim 80 \mu \mathrm{A} / \mathrm{cm}^{2}$, the discharge frequency decreases with the increase of
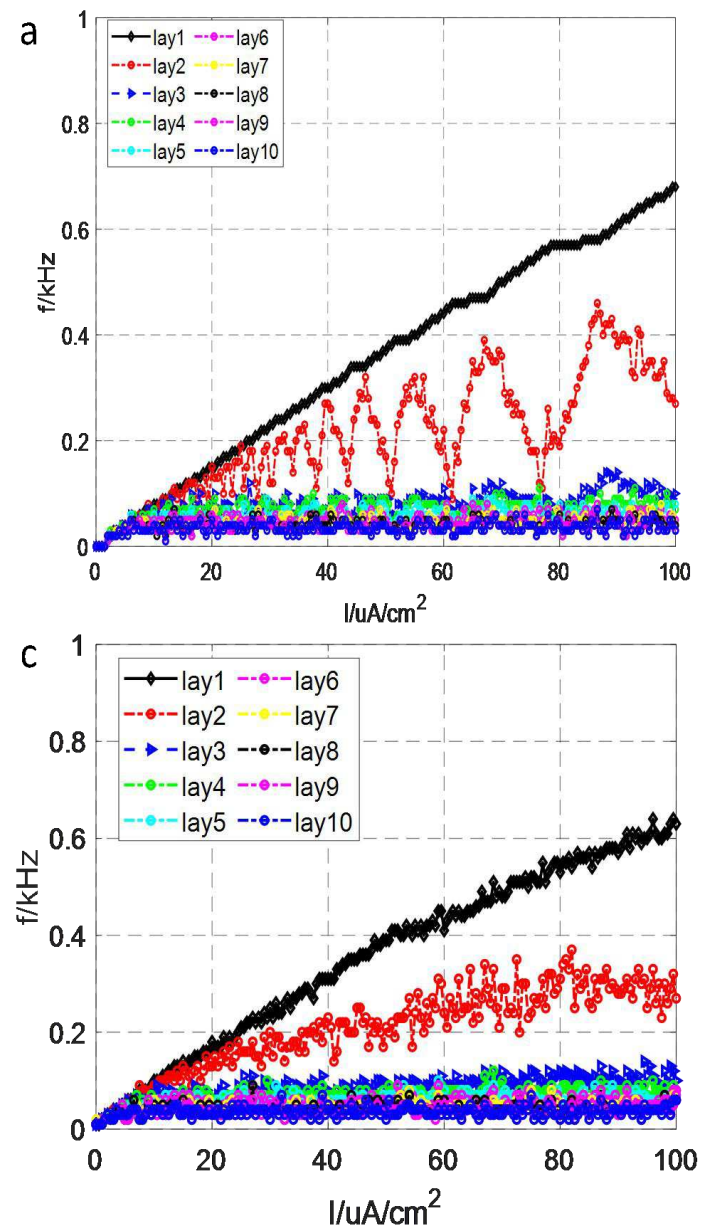

induced current; When it is greater than $80 \mu \mathrm{A} / \mathrm{cm}^{2}$, the discharge frequency of inhibitory neurons in the stimulation layer is basically zero. The change trend of the discharge rate of inhibitory neurons with the induced current in the second to tenth layers of the network is basically the same as that in the stimulation layer, but the amplitude of the discharge rate is smaller than that in the stimulation layer.From the second layer to the tenth layer, the variation trend of the discharge rate of inhibitory neurons with the induced current is basically the same as that of the stimulation layer, but the amplitude of the discharge rate is smaller than that of the stimulation layer.This shows that the effect of magnetic stimulation induced current on inhibitory neurons is stronger than that on excitatory neurons.
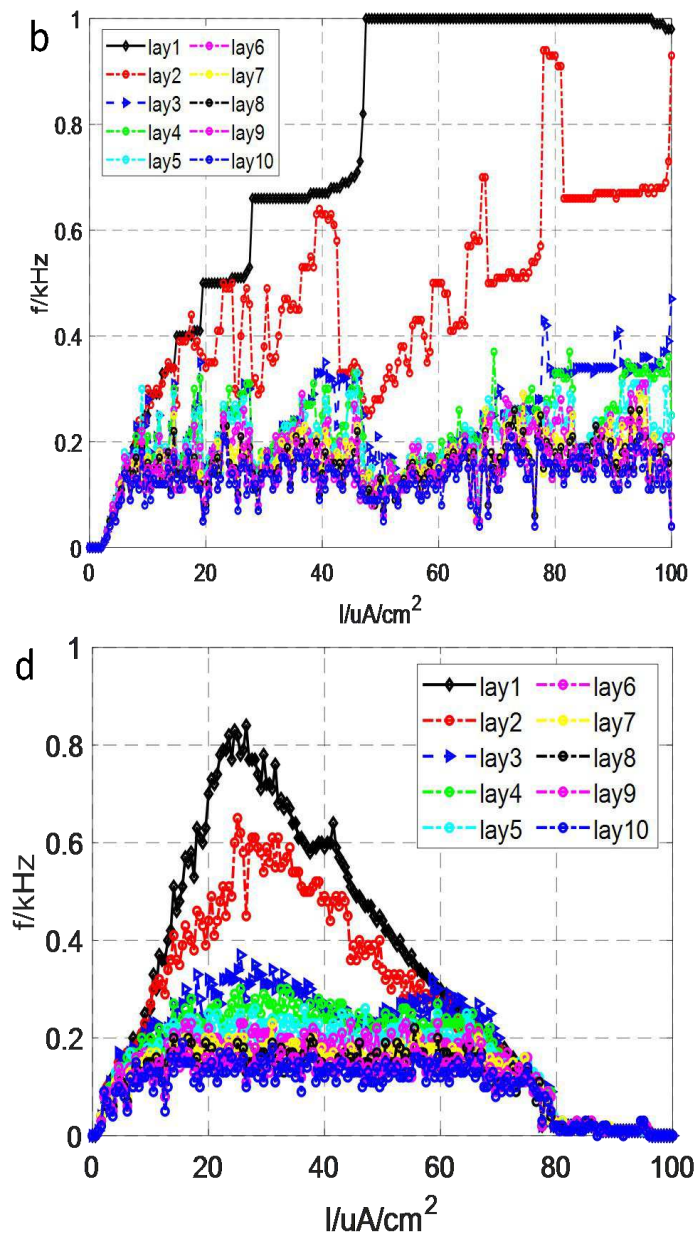

Figure 3.3 Neuron discharge frequency under different induced currents. a)Discharge frequency of excited neurons under TMAES.b)Inhibition of neuronal discharge frequency under TMAES. c)Discharge Frequency of Excited Neurons under TMS.d)Inhibition of neuronal discharge frequency under TMS. 


\subsection{Chemical coupling}

As shown in Fig. 3.4 (a) and Fig. 3.4 (b), the synapses in the feed-forward neural network are connected in the form of chemical coupling. When the induced current is $100 \mu \mathrm{A} / \mathrm{cm}^{2}$ and the coupling strength is 0.8 , the excitability and inhibitory neuronal membrane potential changes in each layer. The discharge of excitatory neurons in the stimulation layer is regular, and the number of spikes decreases with the increase of the number of network layers, and the waveform is missing. The peak-peak interval of inhibitory neurons in the stimulus layer became smaller and subliminal oscillation appeared ; with the increase of the number of network layers, the changes of inhibitory neuron membrane potential in the third layer and later network layer were similar, and the subthreshold oscillation decreased.

In Fig. 3.4 (c) and Fig. 3.4 (d) shows the change of neuron membrane potential in feed-forward neural network under magnetic stimulation.There were discharge spikes in all layers of excitatory neurons within $50 \mathrm{~ms}$ at the beginning of stimulation. The stimulation layer changes from chaotic discharge to regular discharge mode, and the hyperpolarization process is deformed.With the deepening of network layers, the number of discharge spikes of excited neurons decreased. When $\mathrm{t}>40 \mathrm{~ms}$, the excited neurons in the third to tenth layers of the network no longer discharge. All layers of inhibitory neurons produced discharge spikes in the stimulation stage.The discharge mode of inhibitory neurons in the stimulation layer changes alternately from cluster discharge to regular discharge. The discharge mode of inhibitory neurons in the second layer network is peak discharge, but the waveform is distorted.The discharge waveforms from layer 3 to layer 10 are consistent and only two discharge spikes appear.The effect of stimulation on inhibitory neurons is stronger than that on excitatory neurons, and Magnetoacoustic electrical stimulation can stimulate more nerve discharges than magnetic stimulation
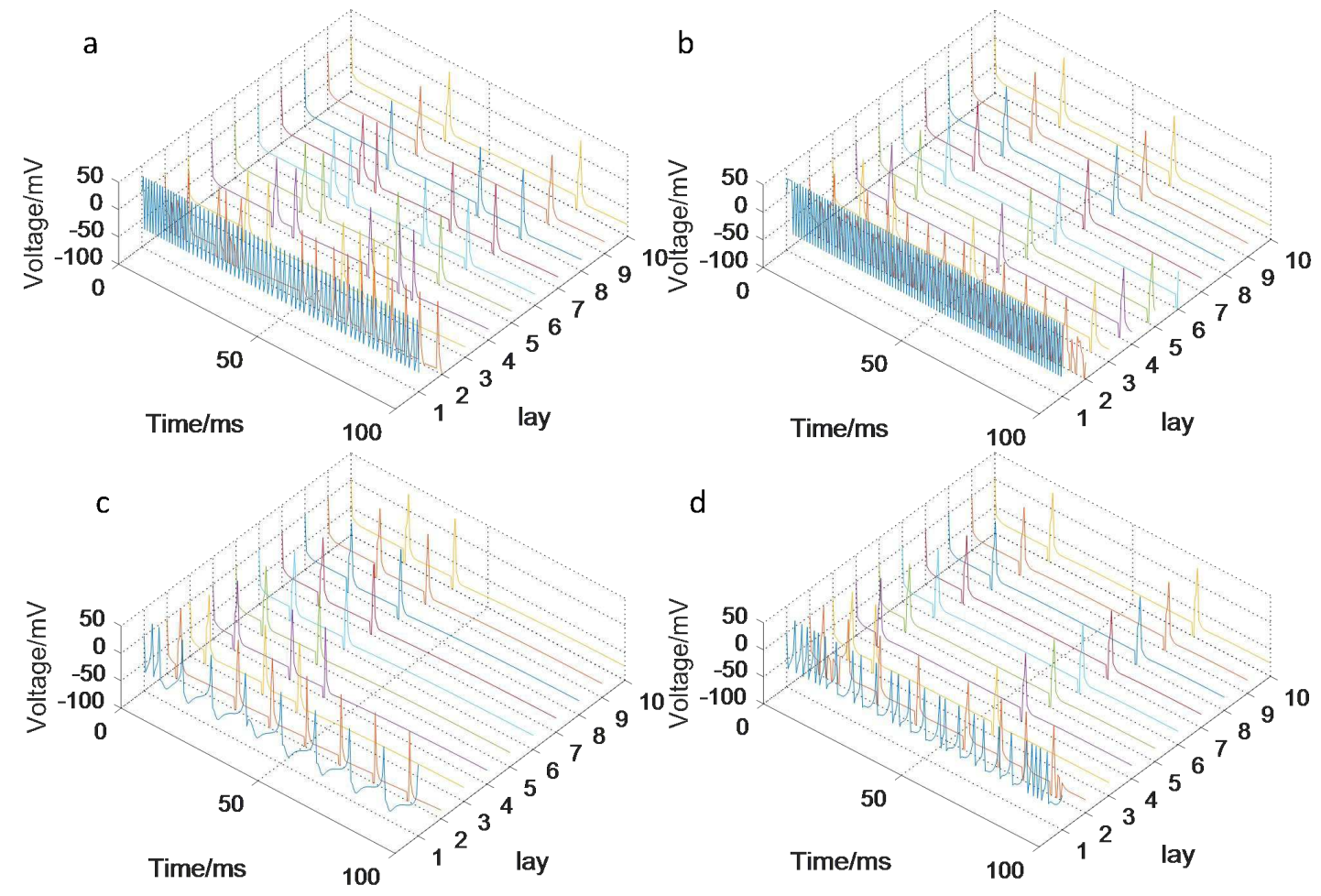

Figure 3.4 Membrane potential of neurons under different external stimuli. a)Membrane potential of excitable neurons under TMAES.b)The membrane potential of inhibitory neurons under TMAES. c)Membrane potential of excitable neurons under TMS.d)The membrane potential of inhibitory neurons under TMS. 
As shown in Figure 3.5 (a) and Figure 3.5 (b), the synapses in the feed-forward neural network are connected in the form of chemical coupling. When the induced current is $100 \mu \mathrm{A} / \mathrm{cm}^{2}$, the discharge frequency of each layer of neurons changes with the degree of chemical coupling. When the chemical coupling intensity was less than 0.6 , the discharge rates of excitatory and inhibitory neurons in each layer were 0 . When the chemical coupling strength is $0.6 \sim$ 0.7 , the discharge rate of excitatory neurons in the neural network increases with the increase of chemical coupling strength, and the discharge rate of inhibitory neurons is still 0 . When the chemical coupling strength was $0.7 \sim 1.0$, with the increase of chemical coupling strength, the discharge frequency of excitatory neurons in each layer decreased 0.02 $\mathrm{kHz}$, and the discharge frequency of inhibitory neurons in each layer increased from 0 to $0.37 \mathrm{kHz}$. It shows that the chemical coupling strength has different effects on neurons of different properties. The discharge frequency of neurons connected by chemical coupling is less than that of neurons connected by electric coupling, which may be due to
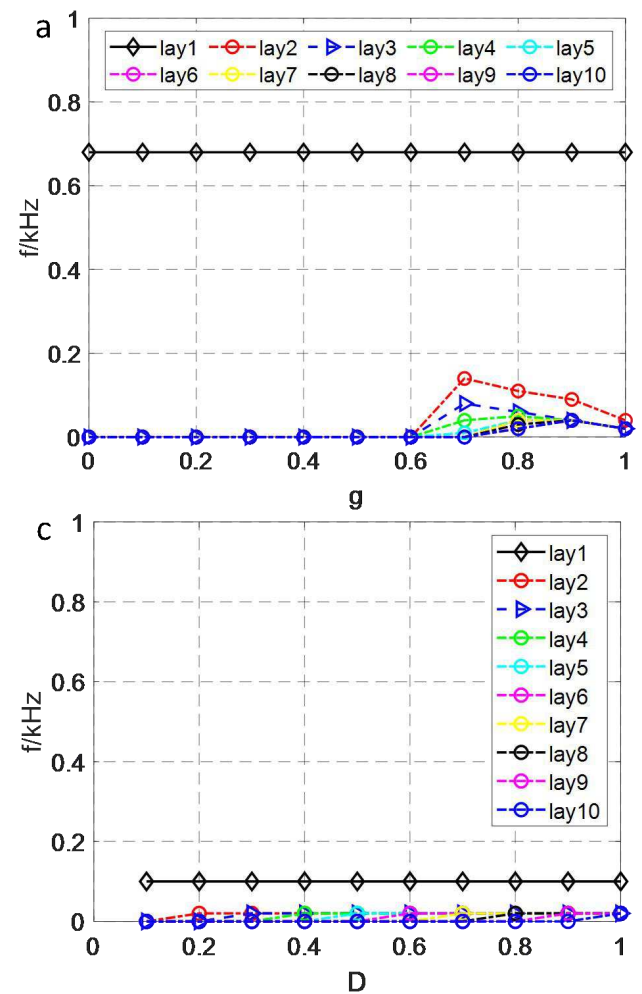

the process of endocytosis and exocytosis of chemical coupling, which slows down the transmission of neural information.In Fig.3.5 (c) and Fig.3.5(d), The discharge rate of excitatory and inhibitory neurons in feed-forward neural network under transcranial magnetic stimulation changes with the increase of coupling degree.The firing rates of excited neurons in each layer remained basically unchanged with the increase of coupling degree, and the firing rates were less than $200 \mathrm{~Hz}$. The firing rate of neurons excited by the stimulating layer was greater than that from the second layer to the tenth layer.The discharge rate of inhibitory neurons in each layer increased with the increase of coupling degree, but the stable value was greater than that of excitatory neurons in each layer.When the electrical coupling degree is 1 , the neurons in each layer of feedforward neural network discharge, and the discharge rate is about $150 \mathrm{~Hz}$. This shows that magnetic stimulation has a stronger effect on inhibitory neurons in the network. The effect of magnetic stimulation on feed-forward neural network is greatly affected by the coupling degree.
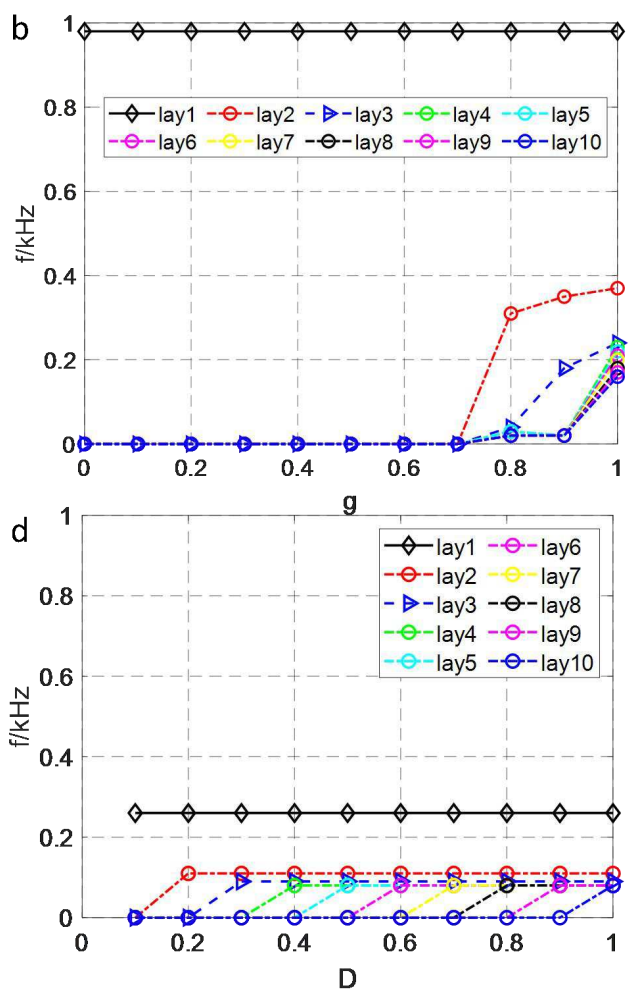

Figure 3.5 Neuron discharge frequency under different external stimuli. a)Excitable neuron discharge frequency under TMAES.b)Discharge frequency of inhibitory neurons under TMAES. c)Excitable neuron discharge frequency under TMS.d)Discharge frequency of inhibitory neurons under TMS. 

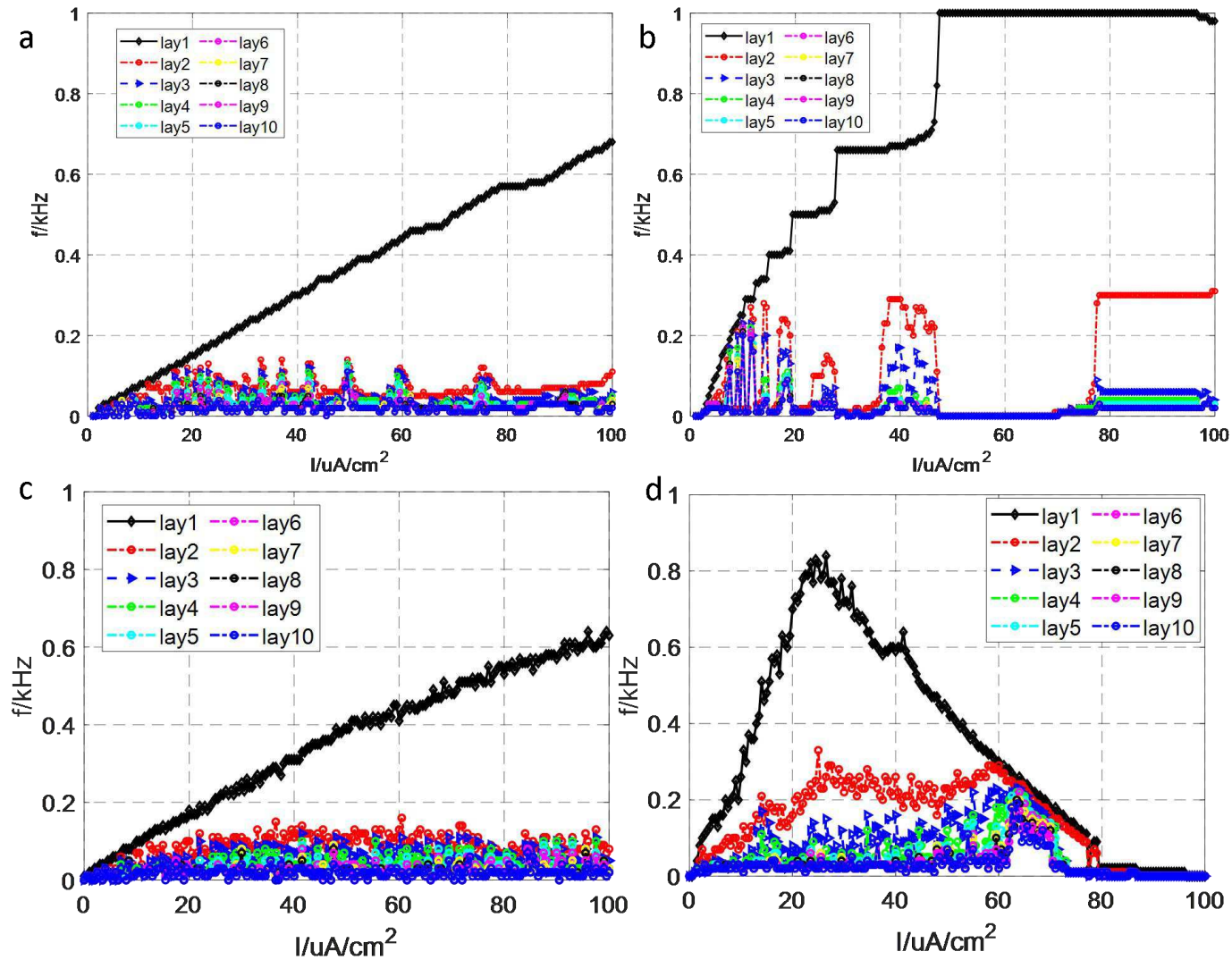

Figure 3.6 Neuron discharge frequency under different external stimuli. a)Discharge frequency of excited neurons in feedforward neural network under TMAES.b)Inhibiting neuron discharge frequency in feedforward neural network under TMAES.

c)Inhibiting neuron discharge frequency in feed-forward neural network under TMS.d)Inhibiting neuron discharge frequency in feed-forward neural network under TMS

As shown in Figure 3.6 (a) and 3.6(b), when the chemical coupling strength is 0.8 , the feed-forward neural network increases the discharge frequency of each layer of neurons with the induced current. With the increase of coupling degree of stimulation layer neural network, the discharge rate of excitatory neurons increased linearly. In the network of the second layer to the tenth layer, the discharge rate fluctuates with the increase of the induced current, and the discharge frequency is below $200 \mathrm{~Hz}$. The discharge rate of neurons inhibited by the stimulation layer increased stepwise when the induced current was less than $45 \mu \mathrm{A} / \mathrm{cm}^{2}$, and the discharge frequency did not change when the induced current was greater than $45 \mu \mathrm{A} / \mathrm{cm}^{2}$. From the second layer to the tenth layer, the change trend of discharge rate of inhibitory neurons was the same. When the induced current was $38 \sim 48 \mu \mathrm{A} / \mathrm{cm}^{2}$, the discharge rate of inhibitory neurons in each layer had a sharp peak, and the larger the number of layers, the smaller the discharge frequency $48 \sim 78 \mu \mathrm{A} / \mathrm{cm}^{2}$, the inhibitory neurons in each layer of the network did not discharge ; when it is greater than $78 \mu \mathrm{A} / \mathrm{cm}^{2}$, the network discharge frequency does not change. The discharge frequency of the second layer of inhibitory neurons is $0.32 \mathrm{kHz}$, and that of the other layers is less than $0.1 \mathrm{kHz}$. In Fig. 3.6c and d show the variation of neuron discharge rate with induced current in each layer of feed-forward neural network under transcranial magnetic stimulation. The discharge rate of excitatory neurons in the stimulation layer increased linearly with the increase of induced current, and the discharge rate from the second layer to the tenth layer decreased successively with the increase of the number of layers, and the discharge rate of excitatory neurons in each layer remained basically unchanged.When the induced current was less than $22 \mu \mathrm{A} / \mathrm{cm}^{2}$, the discharge frequency increased with the increase of induced current, and reached the 
maximum $0.82 \mathrm{kHz}$ when the induced current was $24.5 \mu \mathrm{A} / \mathrm{cm}^{2}$. When the induced current is $24.5 \sim 80 \mu \mathrm{A} / \mathrm{cm}^{2}$, the discharge frequency decreases with the increase of induced current; When it is greater than $80 \mu \mathrm{A} / \mathrm{cm}^{2}$, the discharge frequency of inhibitory neurons in the stimulation layer is basically zero.When the induced current was less than $24.5 \mu \mathrm{A} / \mathrm{cm}^{2}$, the discharge rate waveform showed an upward trend, and the waveform slope was less than that of the stimulation layer; When the induced current is $24.5 \sim 60 \mu \mathrm{A} / \mathrm{cm}^{2}$, the fluctuation of discharge rate is small and stable near $210 \mathrm{~Hz}$. When the induced current is $60 \sim 72 \mu \mathrm{A} / \mathrm{cm}^{2}$, the discharge rate of inhibitory neurons in layer 2 to layer 10 networks decreases to $0 \mathrm{~Hz}$ with the increase of induced current. This shows that the regulation effect of magnetic stimulation induced current on inhibitory neurons is stronger than that on excited neurons.

\section{Conclusion}

In this study, by constructing a ten-layer feed-forward neural network under TMAES and TMS, it analyzed the firing frequency of excited and inhibited neurons under different coupling degrees and induced currents, and concluded that TMAES has more significant adjustment effect.

When the feed-forward neural network is stimulated by TMAES, the firing frequency of excited neurons increases from 0 to $0.4 \mathrm{kHz}$, and the firing frequency of inhibitory neurons varies from 0 to $0.92 \mathrm{kHz}$, which is more susceptible to electrical coupling. Under TMAES, the nerve signal has a discharge spike during the entire stimulation time, but it is only transmitted to the fourth layer; TMS can transmit the stimulation signal to the tenth layer in a short time, but as the stimulation time increases, the deep network nerve signal is missing. This indicates that inhibitory neurons in neural networks are more sensitive to external stimuli and are easily regulated by external nerves. This reflects that inhibitory neurons can transmit more coded information in neuromorphic calculations.

As the degree of electrical coupling increases, the firing frequency of neurons in each layer shows an upward trend under TMAES. When the electrical coupling degree is 0.5 , the second layer of inhibitory neurons reached a maximum discharge frequency of $0.94 \mathrm{kHz}$ under TMAES, and the discharge frequency of inhibitory neurons in the same layer was higher than that of excited neurons. Under TMS neurons begin to discharge only when the coupling degree reaches a certain value, and the discharge frequency is smaller under the same conditions. With the increase of induced current, the discharge frequency of excited neurons of neural network under TMAES presents three waveforms. The discharge frequency of the stimulus layer increases linearly with the rise of the induced current ; the discharge frequency of the second layer network rises in fluctuation, and the fluctuation range is getting larger and larger. The maximum discharge frequency is $0.48 \mathrm{kHz}$. The discharge frequency of other layers is stable below 0.1 $\mathrm{kHz}$. The discharge frequency of inhibitory neurons has three similar waveforms. When the induced current density is less than $48 \mu \mathrm{A} / \mathrm{cm}^{2}$, the stimulation layer shows a step-up trend, and remains unchanged when it is greater than $48 \mu \mathrm{A} / \mathrm{cm}^{2}$. The discharge frequency of the second layer fluctuates greatly with the change of the induced current. The discharge frequency waveform of the other layers is similar to that of the second layer, but smaller than that of the second layer. Under magnetic stimulation, the change trend of discharge frequency of excitatory neurons in the stimulation layer is the same as that of TMAES, and there is a turning threshold for inhibitory neurons. When the electrical coupling degree is greater than $22 \mu \mathrm{A} / \mathrm{cm}^{2}$, the discharge frequency begins to decrease, and the maximum discharge frequency is $0.82 \mathrm{kHz}$. That is, magnetic stimulation has a promoting effect on inhibitory neurons under low stimulation intensity, and high intensity stimulation has an inhibitory effect. When the synapses of neurons are connected by electrical coupling, the signal is transmitted directly through the ion channels on the neural membrane, and the signal transmission speed is faster. To a certain extent, it also reflects the importance of synaptic coupling strength for neural information coding.

The peak-to-peak interval of the chemical coupling neurons under TMAES and TMS is smaller, and the information can be transmitted to the entire 
network layer. However, as the stimulation time increases, the neural signal in the deep network under TMS is no longer transmitted. For inhibitory neurons, magnetic stimulation can transmit signals to the tenth layer during the stimulation period, and the peak-to-peak interval remains unchanged after the third layer, ensuring the accuracy of information transmission and the ability to transmit signals for a long time. When the chemical coupling degree increased to 0.6 , the neural network began to discharge under TMAES. When the coupling degree was 0.7 , the discharge frequency of excited neurons reached the maximum of $0.17 \mathrm{kHz}$, and the inhibitory neuron discharge frequency increased with the increase of the coupling degree. Besides stimulus layer under the magnetic stimulation, other basic layer excited neuron discharge, but the chemical coupling, every 0.1 increase inhibitory neurons discharge layer and add a layer, and discharge frequency is still greater than excited neurons. The mechanism may be related to endocytosis and exocytosis between nerve cells.

With the increase of inductive current, the firing frequency of excitatory neurons in the neural network under the action of TMAES presents two waveforms. The first type is oblique, and the discharge frequency of the stimulus layer increases linearly with the increase of the induced current. The second type is horizontal, and the discharge frequency of the second layer and deep layer network fluctuates slightly with the increase of the induced current and is lower than $0.1 \mathrm{kHz}$. The inhibitory neuronal discharge frequency also showed two types. The first type was ladder type. When the current density was less than $48 \mu \mathrm{A} / \mathrm{cm}^{2}$, the neuronal discharge frequency in the stimulus layer showed a ladder upward trend, and remained unchanged when it was greater than $48 \mu \mathrm{A} / \mathrm{cm}^{2}$. The second type is the peak type, and the discharge frequency of the second layer and deep layer fluctuates greatly with the change of the induced current. At $38 \mu \mathrm{A} / \mathrm{cm}^{2}$ and $78 \mu \mathrm{A} / \mathrm{cm}^{2}$, the discharge frequency of each layer has a steep rise waveform, indicating that when the induced current reaches a certain range, it has a regulatory effect on inhibiting neurons in the neural network. The discharge frequency waveform of excited neurons in the neural network under magnetic stimulation was similar to that under TMAES, but the amplitude was small. The inhibitory neurons in the stimulus layer showed an upward trend when the induction current was less than $24.5 \mu \mathrm{A} / \mathrm{cm}^{2}$, and a downward trend when the induction current was more than $24.5 \mu \mathrm{A} / \mathrm{cm}^{2}$. The discharge frequency of the second layer and deep layer networks was less than $0.3 \mathrm{kHz}$. Magnetic stimulation only had dual regulation effect on the first layer network.

In summary, when the neural network transmits information, the transmission efficiency and excitation degree of deep network are related to the form and coupling strength of synaptic connections. External stimuli can change the efficiency of neural information transmission. Magnetic stimulation improves the depth of information transmission to a certain extent. Induction current can double regulate and inhibit the discharge rate of neurons, and TMS can significantly change the information encoding and transmission of electrochemical coupling network, resulting in one-way improvement.

The results of this paper are helpful to further study the discharge frequency adaptability of the nervous system, explore the signal processing mechanism of the nervous system under the action of external electric field, and provide a theoretical basis for exploring the impact of external electric field on the biological nervous system.It is of positive significance to explore the effect of TMAES on information transmission in neural network, help to deeply understand the modeling of real neural mimicry, and provide some theoretical guidance for the treatment of neurological diseases.

\section{Delclaration of Competing Interest}

The authors declare that they have no known competing financial interests or personal relationships that could have appeared to influence the work reported in this paper.

\section{Acknowledgements}


This work was supported in part by the National Natural Science Foundation of China under Grant No. 51877069. Natural Science Fund Project of Hebei Province under Grant No. E2021202184.

\section{References}

[1] A. Kumar, S. Rotter, A. Aertsen, Spiking activity propagation in neuronal networks: reconciling different perspectives on neural coding, Nat. Rev. Neurosci. 11 (9) (2010) 615-627,https://doi.org/10.1038/n rn2886.

[2] Steinmetz NA, Zatka-Haas P, Carandini M, Harris KD. Distributed coding of choice,action and engagement across the mouse brain, Neuron 576(7786) (2019) 266-301.https://doi.org/10.1038/s41586-0 19-1787-X.

[3] Abbott A, The Extended Mind :The Power of Thinking Outside the Brain, Nature 596 (7871) (2020)181-182. doi :10.1038/d41586-021-02169-3.

[4] Panzeri Stefano, Harvey CD, Piasini Eugenio, Latham Peter E,Fellin Tommaso, Cracking the Neural Code for Sensory Perception by Combining Statistics, Intervention, and Behavior, Neuron 93(3) 4491-507.doi:10.1016/j. neuron.2016.12.036.

[5] Adams, Thomas G JrRippey, Colton SKelly, Alexandra RGold, Brian TPittenger, ChristopherTreatment of obsessive-compulsive disorder with frontopolar multifocal transcranial direct current stimulation and exposure and response prevention: A case Series, Brain stimulation 14 (6) (2021).1431-1433.doi: 10.1016/j.brs.2021.09.008.

[6] Duan.X Q, Yao G, Liu ZL,Cui RJ, Yang $\mathrm{W}$, Mechanisms of transcranial magnetic stimulation treating on post-stroke depression,Frontiers in Human Neuroscience $\quad$ 12(2018) 215 doi:10.3389/fnhum.2018.00215.
[7] Yang HF, Yuan Y,Wang XR,Li X, Closed-Loop Transcranial Ultrasound Stimulation for Real-Time Non-invasive Neuromodulation in vivo . FROINT IN NEURONSCIENCE. 14(445)(2020), doi:10.3389/fnins.2020.00445.

[8] Liu Dan,Zhao Song,Luo Xiaoyuan , Yuan Yi,Unidirectional Synchronization of Hodgkin-Huxley Neurons With Prescribed Performance Under Transcranial Magneto-Acoustical Simulation,Frontiers in neuroscience.13 (2019) 1061. doi:10.3389/fnins.2019. 01061 .

[9] Norton S J. Can Ultrasound be Used to Stimulate Nerve Tissue? Biomedical Engineering Online. 2(2003) (1),http://bi omedical-engineering-online.com/content /2/1/6.

[10] Nadim Farzan, Bucher Dirk. Neuromodulation of neurons and synapses. Current opinion in neurobiology. $29 \quad$ (2014) 48-56. doi:10.1016/j.conb.2014.05.003.

[11] P.-X. Lin, C.-Y. Wang, Z.-X. Wu, Two-fold effects of inhibitory neurons on the onset of synchronization in Izhikevich neuronal networks, Eur. Phys. J. B. 92 (5)(2019) 113.doi:10.11 40/epjb/e2019-100009-2.

[12] A.S. Eteme, C.B. Tabi, A. Mohamadou, iring and synchronization modes in neural network under magnetic stimulation, Commun. Nonlinear Sci. Numer.Simul. 72 (2019) 432-440.doi: 10.1016/j.cnsns.2019.01.004

Tan Hongfang,Wang L iqiang,Interaction of neuronal and network mechanisms on firing propagation in a feedforward network.Eurocomputing. 410 (2020) 93-102.doi:10.1016/j.neucom.2020.05. 088.

[14] Andreev A.V, Frolov NS,Pisarchik A 
N,Hramov A.E, Chimera state in complex networks of bistable Hodgkin-Huxley neurons. PHYSICAL REVIEW E . 100 (2019)2.doi:10.1103/

PhysRevE.100.022224.

[15] Xu Quan,Tan Xiao,Zhu Dong,Chen

Mo,Zhou Jie,Wu Huagan,Synchronous Behavior for Memristive Synapse-

Connected Chay Twin-Neuron Network and Hardware Implementation.

Mathematical Problems In Engineering. 2020.doi:10.1155/2020/8218740.

[16] Tsumoto K, Kitajima H, Yoshinaga T, Aoshinaga T, Aihara K, Kawakami H.Bifurcations in Morris-Lecar Neuron Model.Neurocomputing,69(2005)(4-6):2 93-316.doi:10.1016/j.neucom.2005.03.00 6.

[17] Eugene M. Izhikevich.Simple Model of Spiking Neurons.IEEE Transactions on neural networks.69 (4-6) (2003) 1569-1572.

[18] Xu LF, Li CD, Chen L. Contrastive analysis of neuron model.Actaphysica sinica.65 (24) (2016).doi:10.7498/aps. 65.240701 .

[19] R.S. Snell,Clinical Neuroanatomy, Lippincott Williams \& Wilkins, 2010,pp.35-64.

[20] Njitacke ZT, Doubla IS, Kengne J,

Cheukem A, Coexistence of firing patterns and its control in two neurons coupled through an asymmetric electrical synapse. 30 (2) (2020) .Chaos, doi: 10.10 63/1.5132280.

[21] Cimen Zuehra, Korkmaz Nimet, Altuncu Yasemin, Kilic Recai. Evaluating the effectiveness of several synchronization control methods applying to the electrical -ly and the chemically coupled hindmars h-rose neurons.Biosystems.198 (2020). doi:10.1016/j.biosystems.2020.104284.

[22] Norton S J. Can Ultrasound be Used to Stimulate Nerve Tissue?. Biomedical
Engineering Online, 2 (2003)(1): 6 . http:www.biomedical-engineering-online. com/content $/ \mathrm{a} / 1 / 6$.

[23] J.E. Hall .Guyton and Hall textbook of Medical Physiology E-Book. Elsevier Health Sciences, 2015, pp. 555-562 .

[24] E.R. Kandel , J.H. Schwartz , T.M. Jessell , S.A. Siegelbaum , A.J. Hudspeth , Principles of Neural Science, McGraw-hi 11, New York, 2000, pp. 177-188 . 